\title{
PERFORMANCE ANALYSIS OF MB-OFDM UWB SYSTEM WITH IMPERFECT SYNCHRONIZATION AND INTERSYMBOL INTERFERENCE
}

\author{
Hung-Quoc Lai \\ Department of ECE \\ University of Maryland, \\ College Park, MD 20742 \\ Email: laiquoc@hotmail.com
}

\author{
W. Pam Siriwongpairat \\ Meteor Communications Corporation \\ 22614 66th Ave S. \\ Kent, WA, 98032 \\ Email: PSiriwongpairat@meteorcomm.com
}

\author{
K. J. Ray Liu \\ Department of ECE \\ University of Maryland, \\ College Park, MD 20742 \\ Email: kjrliu@eng.umd.edu
}

\begin{abstract}
This paper provides performance analysis of multiband OFDM UWB system that takes into consideration the effect of intersymbol interference and imperfect frequency and timing synchronization. The system performance is analyzed under UWB multipath fading channels, as specified in the IEEE 802.15.3a channel standard. Average signal-to-noise ratio is first derived for the system. Then an approximation technique is employed to obtain a closed-form average bit error probability that provides a profound understanding of the performance of the multiband OFDM UWB system. Simulation results validate the theoretical analysis.

Index Terms - Ultra-wideband, OFDM, bit error probability, frequency and timing synchronization, intersymbol interference
\end{abstract}

\section{INTRODUCTION}

Ultra-wideband (UWB) has emerged as a technology for shortrange, high data-rate communications. To exploit the unlicensed 7.5GHz bandwidth $(3.1-10.6 \mathrm{GHz})$, two technical approaches have mainly been proposed: direct-sequence UWB relating to single-band systems and multiband UWB. The dominant candidate of the multiband approach employs orthogonal frequency division multiplexing (OFDM) technique, the so-called MB-OFDM UWB [1]. In this paper, we consider MB-OFDM UWB.

The system supports 10 data rates from $53.3 \mathrm{MHz}$ to $480 \mathrm{MHz}$ [1], which are grouped into three data-rate modes, namely high-rate, middle-rate, and low-rate based on overall spreading gain factors of 1,2, and 4. Four UWB standard channel models, denoted as CM1, CM2, CM3, and CM4 specified in the IEEE 802.15.3a [2] are derived from S-V model [3]. The channel models are characterized by cluster and ray arrival rates and decay factors.

Performance analysis of MB-OFDM UWB system has been an area of considerable interest. A number of system performances has been published in the literature (see [4], [5], [6]). All the existing work assumed perfect frequency and timing synchronization. The channel multipath delays were also assumed to fit inside OFDM cyclic prefix and hence the system would not suffer intersymbol interference (ISI). In practice, the delays, however, can exceed the length of the

This work was supported in part by U.S. Army RDECOM CERDEC. cyclic prefix, causing ISI. Moreover, OFDM technique is also sensitive to imperfect frequency and timing synchronization.

This paper thoroughly analyzes the performance of MBOFDM UWB system in UWB channel models with ISI and imperfect synchronization. Average signal-to-noise ratio (SNR) is first derived for the system. Then an approximation technique is employed to obtain a closed-form average bit error probability that provides a profound understanding of the performance of the multiband OFDM UWB system. The simulation results validate the theoretical analysis.

\section{CHANNEL AND SYSTEM MODELS}

\subsection{Channel Model}

UWB standard channel models specified in the IEEE 802.15.3a [2] are derived from $\mathrm{S}-\mathrm{V}$ model [3]. We can rewrite channel impulse response for $\mathrm{CMl}$ as

$$
\begin{aligned}
h(t)= & \alpha_{0,0} \delta(t)+\sum_{k=1}^{K} \alpha_{k, 0} \delta\left(t-\tau_{k, 0}\right)+\sum_{l=1}^{L} \alpha_{0, l} \delta\left(t-T_{l}\right) \\
& +\sum_{l=1}^{L} \sum_{k=1}^{K} \alpha_{k, l} \delta\left(t-T_{l}-\tau_{k, l}\right)
\end{aligned}
$$

where $\alpha_{k, l}$ 's are multipath gain coefficients, $T_{l}$ and $\tau_{k, l}$ are the delay of the $l^{\text {th }}$ cluster and the $k^{\text {th }}$ ray in the $l^{\text {th }}$ cluster. The cluster and ray arrival times $T_{l}$ 's and $\tau_{k, l}$ 's are modelled as time of arrivals in Poisson processes with rate $\Lambda$ and $\lambda$ (where $\lambda>\Lambda$ ) [2], respectively. Multipath gain coefficients $\alpha_{k, l}$ 's are modelled as statistically independent, zero-mean, complex Gaussian random variables whose variance is [2]

$$
\Omega_{k, l}=E\left\{\left|\alpha_{k, l}\right|^{2} \mid T_{l}, \tau_{k, l}\right\}=\Omega_{0,0} e^{-\frac{T_{l}}{\Gamma}-\frac{\tau_{k, l}}{\gamma}}
$$

where $\Gamma$ and $\gamma$ are cluster and ray decay factors, respectively. Channel impulse response for CM2, CM3, and CM4 takes the form of Eq. (1) without the first two terms.

\subsection{Signal Model}

The received signal is $r(t)=\sum_{i=-\infty}^{\infty} y_{i}(t)+n(t)$ where

$$
\begin{aligned}
y_{i}(t) & =\frac{1}{T_{S}} \sum_{n=0}^{N-1} c_{n, i} \sum_{l=0}^{L} \sum_{k=0}^{K} \alpha_{k, l} \\
& \times g\left(t-i T_{S}^{\prime}-T_{l}-\tau_{k, l}\right) e^{\frac{j 2 \pi n\left(t-i T_{S}^{\prime}-T_{l}-\tau_{k, l}\right)}{T_{S}}}
\end{aligned}
$$

is the channel response corresponding to OFDM symbol $x_{i}$ and $n(t)$ is additive white Gaussian noise (AWGN). In Eq. 
(3), $T_{S}$ and $T_{S}^{\prime}$ are the durations of the useful and transmitted OFDM symbol, respectively. At the receiver, we consider imperfect frequency and timing synchronization with a carrier-frequency offset $\Delta f$ and a timing error $\tau$. Accordingly, the demodulated signal $\hat{c}_{m, i}$ is

$$
\hat{c}_{m, i}=\int_{i T_{S}^{\prime}+T_{C}-\tau}^{i T_{S}^{\prime}+T_{C}+T_{S}-\tau} r(t) e^{-j 2 \pi\left(f_{t, m}+\Delta f\right)\left(t-i T_{S}^{\prime}\right)} \mathrm{dt}
$$

where $f_{t, m}$ is the transmitter carrier frequency corresponding to the subcarrier $m$. Let $\varepsilon=\frac{\Delta f}{1 / T_{S}}=\Delta f T_{S}$ be relative carrier-frequency offset. Then it can be shown that

$$
\hat{c}_{m, i}=c_{m, i} H_{m}+\hat{c}_{m, i}^{I C I}+\hat{c}_{m, i}^{I S I}+n_{m, i}
$$

where

$$
\begin{aligned}
& H_{m}=w^{\varepsilon T_{C}} w^{-(m+\varepsilon) \tau} \\
& \times\left[\sum_{l=0}^{l_{0}} \sum_{k=0}^{K} \alpha_{k, l} w^{m X_{k, l}} \frac{\left(e^{-j 2 \pi \varepsilon} w^{\varepsilon X_{k, l}}-1\right)}{-j 2 \pi \varepsilon}\right. \\
& \left.+\sum_{l=l_{0}+1}^{L} \sum_{k=0}^{K} \alpha_{k, l} w^{m X_{k, l}} \frac{\left(e^{-j 2 \pi \varepsilon}-w^{\varepsilon X_{k, l}}\right)}{-j 2 \pi \varepsilon}\right] \\
& \hat{c}_{m, i}^{I C I}=\sum_{n \neq m} c_{n, i} w^{-(n-m-\varepsilon) T_{C}} w^{-(m+\varepsilon) \tau} \\
& \times\left[\sum_{l=0}^{l_{0}} \sum_{k=0}^{K} \alpha_{k, l} \frac{\left(e^{-j 2 \pi \varepsilon} w^{(m+\varepsilon) X_{k, l}}-w^{n X_{k, l}}\right)}{j 2 \pi(n-m-\varepsilon)}\right. \\
& \left.+\sum_{l=l_{0}+1}^{L} \sum_{k=0}^{K} \alpha_{k, l} \frac{\left(e^{-j 2 \pi \varepsilon} w^{n X_{k, l}}-w^{(m+\varepsilon) X_{k, l}}\right)}{j 2 \pi(n-m-\varepsilon)}\right] \\
& \hat{c}_{m, i}^{I S I}=\sum_{n=0}^{N-1} c_{n, i-1} w^{-(n-m-\varepsilon) T_{C}} w^{-(m+\varepsilon) \tau} \\
& \times \sum_{l=l_{0}+1}^{L} \sum_{k=0}^{K} \frac{\alpha_{k, l}}{j 2 \pi(n-m-\varepsilon)} \\
& \times\left(w^{(m+\varepsilon)\left(X_{k, l}-T_{C}-T_{G}\right)}-w^{n\left(X_{k, l}-T_{C}-T_{G}\right)}\right)
\end{aligned}
$$

are fading term, intercarrier interference (ICI), and ISI, respectively and $n_{m, i} \sim C N\left(0, N_{0}\right)$ is AWGN. In the above equations, we have defined $X_{k, l}=T_{l}+\tau_{k, l}+\tau$ and $w=$ $e^{\frac{-j 2 \pi}{T_{S}}}$ for notational convenience.

The following assumptions are employed in this performance analysis.

Assumption 1: $\tau \in\left(-T_{C}, T_{C}\right)$. For large timing error $\tau$, the performance possesses very high error probability and hence needs not to be considered.

Assumption 2: $T_{l}+\tau_{k, l} \leq T_{S}$ for all $k, l$. Through the generation of the channel, no delay is larger than the symbol duration $T_{S}$.

Assumption 3: Let $X=T_{l}+\tau_{k, l}-T$ and $l_{0}=\lfloor\Lambda T\rfloor$ for a deterministic time $T$ and cluster arrival rate $\Lambda$. $\lfloor$.$\rfloor rep-$ resents floor function. $l_{0}$ represents the average number of

cluster arrivals at time $T$. Then $X>0 \Leftrightarrow l \geq l_{0}+1$. This assumption implies that if $T_{l}<T$, then all rays in the $l^{\text {th }}$ cluster arrive assumedly at a time less than $T$; in other words, $T_{l}+\tau_{k, l}<T \forall k$.

Assumption 4: Transmitted symbols $c_{n, i}$ 's are independent and identically distributed (i.i.d.) with symbol energy $E_{s}$. Since two bits form a quadrature phase-shift keying (QPSK) symbol, $E_{s}=2 E_{b}$ where $E_{b}$ is the bit energy.

Assumption 5: The channel, the transmitted symbols, and AWGN are mutually independent.

The assumptions simplify the derivation process; however, they still maintain the nature of the problem.

\section{AVERAGE SIGNAL-TO-NOISE RATIO}

The average SNR per QPSK symbol can be defined as

$$
\overline{\gamma_{s}}(\varepsilon, \tau) \triangleq \frac{E_{s} \sigma_{H}^{2}}{\sigma_{C}^{2}+\sigma_{S}^{2}+N_{0}}
$$

where $\sigma_{H}^{2}, \sigma_{C}^{2}$, and $\sigma_{S}^{2}$ are variances of fading term, ICI, and ISI, respectively, $E_{s}$ is symbol energy, and $N_{0}$ is AWGN variance. Since the energy per bit $E_{b}=\frac{1}{2} E_{s}$, the average SNR per bit $\overline{\gamma_{b}}(\varepsilon, \tau)=\frac{1}{2} \overline{\gamma_{s}}(\varepsilon, \tau)$. We will use $\overline{\gamma_{b}}(\varepsilon, \tau)$ to evaluate the system performance.

Because transmitted symbols $c_{n, i}$ 's and multipath gain coefficients $\alpha_{k, l}$ 's are zero-mean, $H_{m}, \hat{c}_{m, i}^{I C I}$, and $\hat{c}_{m, i}^{I S I}$ are also zero-mean. Thus $\sigma_{H}^{2}=E\left\{\left|H_{m}\right|^{2}\right\}, \sigma_{C}^{2}=E\left\{\left|\hat{c}_{m, i}^{I C I}\right|^{2}\right\}$, and $\sigma_{H}^{2}=E\left\{\left|\hat{c}_{m, i}^{I S I}\right|^{2}\right\}$. Using Ass. 4 and 5 and Eq. (2) for multipath gain coefficients $\alpha_{k, l}$, we are able to show that

$$
\begin{aligned}
& \sigma_{H}^{2}=\frac{1}{4 \pi^{2} \varepsilon^{2}} \sum_{l=0}^{l_{0}} \sum_{k=0}^{K} E\left\{\Omega_{0,0} e^{-\frac{T_{l}}{\Gamma}-\frac{\tau_{k, l}}{\gamma}}\right. \\
& \left.\times \quad\left[2-\left(e^{-j 2 \pi \varepsilon} w^{\varepsilon X_{k, l}}+e^{j 2 \pi \varepsilon} w^{-\varepsilon X_{k, l}}\right)\right]\right\} \\
& +\frac{1}{4 \pi^{2} \varepsilon^{2}} \sum_{l=l_{0}+1}^{L} \sum_{k=0}^{K} E\left\{\Omega_{0,0} e^{-\frac{T_{l}}{\Gamma}-\frac{\tau_{k, l}}{\gamma}}\right. \\
& \left.\times \quad\left[2-\left(e^{-j 2 \pi \varepsilon} w^{-\varepsilon X_{k, l}}+e^{j 2 \pi \varepsilon} w^{\varepsilon X_{k, l}}\right)\right]\right\} \\
& \sigma_{C}^{2}=E_{s} \sum_{n \neq m} \frac{1}{4 \pi^{2}(n-m-\varepsilon)^{2}}\left[\sum _ { l = 0 } ^ { l _ { 0 } } \sum _ { k = 0 } ^ { K } E \left\{\Omega_{0,0}\right.\right. \\
& \times \quad e^{-\frac{T_{l}}{\Gamma}-\frac{\tau_{k, l}}{\gamma}}\left[2-\left(e^{-j 2 \pi \varepsilon} w^{-(n-m-\varepsilon) X_{k, l}}\right.\right. \\
& \left.\left.+\quad e^{j 2 \pi \varepsilon} w^{\left.(n-m-\varepsilon) X_{k, l}\right)}\right]\right\}+\sum_{l=l_{0}+1}^{L} \sum_{k=0}^{K} E\left\{\Omega_{0,0}\right. \\
& \times e^{-\frac{T_{l}}{\Gamma}-\frac{\tau_{k, l}}{\gamma}}\left[2-\left(e^{-j 2 \pi \varepsilon} w^{(n-m-\varepsilon) X_{k, l}}\right.\right. \\
& \left.\left.\left.\left.+\quad e^{j 2 \pi \varepsilon} w^{-(n-m-\varepsilon) X_{k, l}}\right)\right]\right\}\right] \\
& \sigma_{S}^{2}=E_{s} \sum_{n=0}^{N-1} \frac{1}{4 \pi^{2}(n-m-\varepsilon)^{2}} \sum_{l=l_{0}+1}^{L} \sum_{k=0}^{K} E\left\{\Omega_{0,0}\right. \\
& \times \quad e^{-\frac{T_{l}}{\Gamma}-\frac{\tau_{k, l}}{\gamma}}\left[2-\left(w^{(n-m-\varepsilon)\left(X_{k, b}-T_{C}-T_{G}\right)}\right.\right.
\end{aligned}
$$




$$
\left.\left.\left.+\quad w^{-(n-m-\varepsilon)\left(X_{k, l}-T_{C}-T_{G}\right)}\right)\right]\right\} .
$$

To evaluate these variances, we first separate the expectations in term of $T_{l}$ and $\tau_{k, l}$ since these random variables are statistically independent. Then we employ the moments of these random variables for the expectations to obtain the expressions of these quantities. Note that $T_{l}$ and $\tau_{k, l}$ are $l-$ and $k$-Erlang random variables. Due to the space limitation, the derivation is not presented in this paper; however, it will be provided if required.

\section{AVERAGE BIT ERROR PROBABILITY}

Let $z_{m} \triangleq \hat{c}_{m, i}^{I C I}+\hat{c}_{m, i}^{I S I}+n_{m, i}$. Then Eq. (5) can be rewritten as $\hat{c}_{m, i}=c_{m, i} H_{m}+z_{m}$. We model $\hat{c}_{m, i}^{I C I}$ and $\hat{c}_{m, i}^{I S I}$ as Gaussian random variables whose mean is zero and variance is $\sigma_{C}^{2}$ and $\sigma_{S}^{2}$, respectively. Consequently, $z_{m} \sim C N\left(0, \sigma_{Z}^{2}\right)$ where $\sigma_{Z}^{2}=\sigma_{C}^{2}+\sigma_{S}^{2}+N_{0}$.

Ten data rates in the UWB system are grouped into three data-rate modes based on overall spreading gain factors of 1 , 2, or 4 [1]. The three cases share the same receiving model: $\hat{\mathbf{c}}=c_{m, i} \mathbf{h}+\mathbf{z}$ where $\hat{\mathbf{c}}$ and $\mathbf{h}$ are vectors comprising demodulated signals $\hat{c}_{m, i}$ and fading terms $H_{m}$ associated with $\hat{c}_{m, i}$, respectively while $\mathbf{z} \sim C N\left(0, \sigma_{Z}^{2} \mathbb{I}\right)$ is the noise vector.

The average bit error probability can be shown as

$$
P_{b} \approx p^{m} \sum_{k=0}^{m-1}\left(\begin{array}{c}
m-1+k \\
k
\end{array}\right)(1-p)^{k}
$$

where $m$ is the gain factor and $p=\frac{1}{2}\left(1-\sqrt{\frac{\overline{\gamma_{\rho}}}{1+\overline{\gamma_{\rho}}}}\right)$ with $\overline{\gamma_{\rho}} \triangleq E\{\rho\}$ corresponding to $m=1$ and

$$
\rho=\|\mathbf{h}\|^{2} \frac{E_{b}}{\sigma_{Z}^{2}} .
$$

Eq. (13) is resulted from the fact that $\rho$ is approximately chi-square distributed with $2 m$ degrees of freedom. In what follows, we will present the proof for the case of $m=1$. The same calculation steps can be used to get the results for other cases.

In case of $m=1, \hat{\mathbf{c}}=\hat{c}_{m, i}, \mathbf{h}=H_{m}$, and $\mathbf{z}=z$. Thus $\rho=\frac{E_{b}}{\sigma_{Z}^{2}}\left|H_{m}\right|^{2}$. From Eq. (6), we rewrite the fading term as

$$
H_{m}=\frac{1}{-j 2 \pi \varepsilon} w^{\varepsilon T_{C}} w^{-(m+\varepsilon) \tau} \mathbf{w}^{H} \mathbb{T} \mathbf{a}
$$

where $\mathbf{w}=\left[w^{m\left(T_{0}+\tau_{0,0}+\tau\right)}, w^{m\left(T_{0}+\tau_{0,1}+\tau\right)}, \ldots\right.$, $\left.w^{m\left(T_{L}+\tau_{K, L}+\tau\right)}\right]^{T}, \mathbb{T}=\operatorname{diag}\left(e^{-j 2 \pi \varepsilon} w^{\varepsilon\left(T_{0}+\tau_{0,0}+\tau\right)}-1\right.$ $, \ldots, e^{-j 2 \pi \varepsilon} w^{\varepsilon\left(T_{l_{0}}+\tau_{K, l_{0}}+\tau\right)}-1, e^{-j 2 \pi \varepsilon} w^{\varepsilon\left(T_{l_{0}+1}+\tau_{0, l_{0}+1}+\tau\right)}$ $\left.-1, \ldots, e^{-j 2 \pi \varepsilon} w^{\varepsilon\left(T_{L}+\tau_{K, L}+\tau\right)}-1\right)$ and $\mathbf{a}=\left[\alpha_{0,0}, \alpha_{0,1}, \ldots\right.$, $\left.\alpha_{K, L}\right]^{T}$. Because $\alpha_{k, l} \sim C N\left(0, \Omega_{k, l}\right), \mathbf{a}=\Omega^{\frac{1}{2}} \mathbf{b}$ where $\boldsymbol{\Omega}^{\frac{1}{2}} \boldsymbol{\Omega}^{\frac{1}{2}}=\boldsymbol{\Omega}=\operatorname{diag}\left(\Omega_{0,0}, \Omega_{0,1}, \ldots, \Omega_{K, L}\right)$ and $\mathbf{b}=\left[\beta_{0,0}^{\prime}\right.$, $\left.\beta_{0,1}^{\prime}, \ldots, \beta_{K, L}^{\prime}\right]^{T}$ where $\beta_{k, l}^{\prime} \sim C N(0,1)$. Consequently,

$$
\rho=\frac{E_{b}}{\sigma_{Z}^{2}} \frac{1}{4 \pi^{2} \varepsilon^{2}} \mathbf{b}^{H} \boldsymbol{\Omega}^{\frac{1}{2}} \mathbb{T} \mathbf{w} \mathbf{w}^{H} \mathbb{T} \boldsymbol{\Omega}^{\frac{1}{2}} \mathbf{b} .
$$

Let us define $\Psi=\Omega^{\frac{1}{2}} \mathbb{T} \mathbf{w} \mathbf{w}^{H} \mathbb{T} \Omega^{\frac{1}{2}}$. Since $\Psi$ is a nonnegative definite Hermitian matrix, it can be expressed as $\boldsymbol{\Psi}=\mathbb{V} \boldsymbol{\Lambda} \mathbb{V}^{H}$ where $\boldsymbol{\Lambda}$ is an eigenvalue matrix and $\mathbb{V}$ is a unitary matrix. Since $\operatorname{rank}(\boldsymbol{\Psi}) \leq \min \left\{\operatorname{rank}\left(\boldsymbol{\Omega}^{\frac{1}{2}}\right), \operatorname{rank}(\mathbb{T})\right.$, $\operatorname{rank}(\mathbf{w})\}$ where $\operatorname{rank}\left(\boldsymbol{\Omega}^{\frac{1}{2}}\right)=\operatorname{rank}(\mathbb{T})=(K+1)(L+1)$ and $\operatorname{rank}(\mathbf{w})=1$, there exists in $\Lambda$ only one nonzero eigenvalue, which can be evaluated as $\operatorname{eig}(\boldsymbol{\Psi})=D$, and hence

$$
\rho=\frac{E_{b}}{\sigma_{Z}^{2}} \frac{1}{4 \pi^{2} \varepsilon^{2}} D|\beta|^{2}
$$

where $\beta \sim C N(0,1)$ and

$$
\begin{aligned}
D & =\sum_{l=0}^{l_{0}} \sum_{k=0}^{K} \Omega_{0,0} e^{-\frac{T_{l}}{\Gamma}-\frac{\tau_{k, l}}{\gamma}}\left[2-\left(e^{-j 2 \pi \varepsilon} w^{\varepsilon X_{k, l}}\right.\right. \\
& \left.\left.+e^{j 2 \pi \varepsilon} w^{-\varepsilon X_{k, l}}\right)\right]+\sum_{l=l_{0}+1}^{L} \sum_{k=0}^{K} \Omega_{0,0} e^{-\frac{T_{l}}{\Gamma}-\frac{\tau_{k, l}}{\gamma}} \\
& \times\left[2-\left(e^{-j 2 \pi \varepsilon} w^{-\varepsilon X_{k, l}}+e^{j 2 \pi \varepsilon} w^{\varepsilon X_{k, l}}\right)\right]
\end{aligned}
$$

Eq. (17) reveals that $\rho$ is not a chi-square random variable with two degrees of freedom as in the case of Rayleigh fading channel because of the $T_{l}$ and $\tau_{k, l}$. To solve the problem, we employ the approximation approach in [6]. From Eq. (14), $\rho$ has a quadratic form, and thus can be rewritten as [7]

$$
\rho \approx \frac{E_{b}}{\sigma_{Z}^{2}} \sum_{s=1}^{S} \operatorname{eig}_{s}(\boldsymbol{\Phi})\left|\mu_{s}\right|^{2}
$$

where $\mu_{s} \sim C N(0,1)$ and $S$ is the rank of matrix $\Phi \triangleq$ $E\left\{\mathbf{h h}^{H}\right\}$. In case of gain factor $m=1, \boldsymbol{\Phi}=\sigma_{H}^{2}$, which is the variance of the fading term. Consequently,

$$
\rho \approx \frac{E_{b} \sigma_{H}^{2}}{\sigma_{Z}^{2}}|\mu|^{2} .
$$

Since $\mu \sim C N(0,1), \rho$ approximately is chi-square-distributed with two degrees of freedom. Eq. (20) also reveals that the expectation of $\rho: \overline{\gamma_{\rho}}=\overline{\gamma_{b}}(\varepsilon, \tau)$, the average SNR per bit. Substituting this expectation into Eq. (13), we get the average bit error probability for this case.

\section{NUMERICAL AND SIMULATED RESULTS}

We perform simulations with the OFDM system and the channel characteristics following [1]. For demonstration purpose, we present the numerical results in CM2 and the simulation result in CM1. In Figure 1, we plot the average bit error probability of the OFDM system against the SNR per bit for the low-rate mode and various timing synchronization errors to illustrate the OFDM system performance in the imperfect timing synchronization. In the figure, $T=\frac{T_{S}}{N}$ where $T_{S}$ is the useful OFDM duration and $N$ is the number of OFDM subcarriers. The figure reveals that positive timing errors always worsen the system performance while small negative timing errors can improve it. In the figure, the $3 T$ line is above the $0 T$ one while the lines associated with negative $T$ 


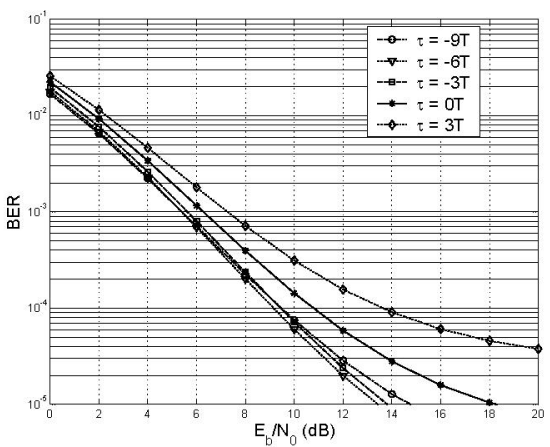

Fig. 1. Performance of MB-OFDM UWB system with timing synchronization errors.

are below the $0 T$ one. Also for negative $\tau$, the increment of its magnitude reduces the performance improvement. In the figure, among the lines with negative $T$, the $-6 T$ line has the best performance.

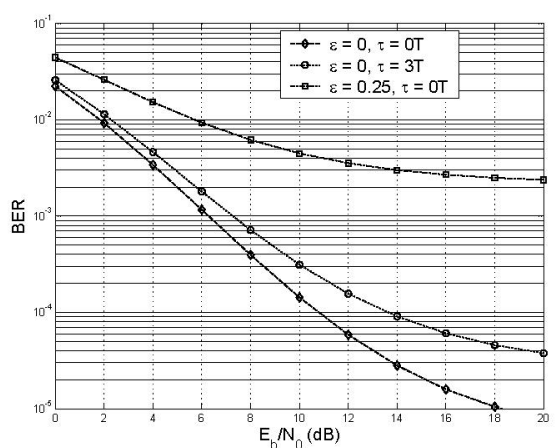

Fig. 2. Performance of MB-OFDM UWB system: timing error vs. frequency error.

In Figure 2, we compare the system performance between timing synchronization and frequency synchronization. The figure reveals clearly that frequency synchronization is more important than timing synchronization. The performance of the system with frequency synchronization error degrades much faster than that of the system with time synchronization error does.

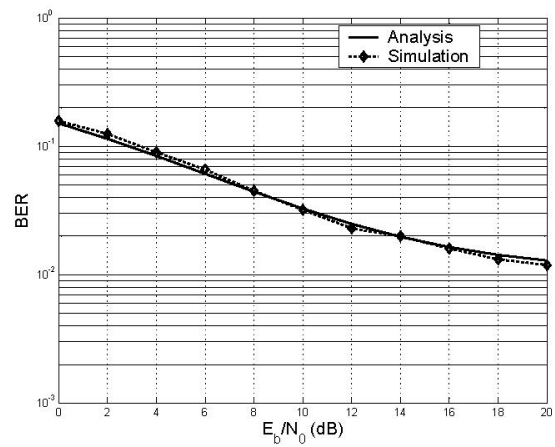

Fig. 3. Performance of MB-OFDM UWB system: simulation vs. analysis.

The simulation result is plotted together with the numerical result in Figure 3. So far, we are able to obtain only the simulation average bit error probability for the OFDM system for the high-rate mode in the case of channel model $\mathrm{CMl}$ and perfect synchronization. The simulation consumes an enormous amount of time. The reason is due to the high average number of channel multipath delays. The numbers of delays for CM1, CM2, CM3, and CM4 are 295, 765, 1460 , and 3930 in average, respectively. In addition, the computation of the demodulated signal $\hat{c}_{m, i}$ requires the integration of the received signal $r(t)$, as we see in Eq. (4). The integration is computed numerically. The whole process really consumes a great amount of time. For channel models CM2, $\mathrm{CM} 3$, and CM4, the computer cannot even handle it.

Although we have the limitation to the computing resources, Figure 3 shows that the simulation result matches the numerical result very well. The simulation validates our performance analysis.

\section{CONCLUSIONS}

We provide the performance analysis of MB-OFDM UWB system in the four IEEE 802.15.3a channel models under imperfect frequency and timing synchronization and ISI. The results show that small negative timing synchronization error can improve the system performance. In addition, frequency synchronization is more important than timing synchronization since frequency error degrades the system performance much more than timing error does. Although we are able to obtain only the simulation result for the high-rate mode in $\mathrm{CMl}$ and perfect frequency and timing synchronization, the simulation validates our theoretical analysis.

\section{REFERENCES}

[1] A. Batra et al., "Multi-band OFDM physical layer proposal for IEEE 802.15 task group 3a," tech. rep., IEEE P802.1503/268r3, March 2004.

[2] J. Foerster et al., "Channel modeling sub-committer report final," tech. rep., IEEE P802.15-02/368r5-SG3a, July 2004.

[3] A. Saleh and R. Valenzuela, "A statistical model for indoor multipath propagation," IEEE J. Select. Areas Commun., vol. 5, pp. 128-137, Feb. 1987.

[4] O. Shin et al., "Performance evaluation of MB-OFDM and DS-UWB systems for wireless personal area networks," IEEE ICUWB'05, pp. 214-219, September 2005.

[5] C. Snow et al., "Performance analysis of multiband OFDM for UWB communication," IEEE ICC'05, vol. 4, pp. 2573-2578, May 2005.

[6] W. P. Siriwongpairat, W. Su, and K. J. R. Liu, "Performance characterization of multiband UWB communication systems using Poisson cluster arriving fading paths," IEEE JSAC, Special Issue on Ultra Wideband Wireless Communications - Theory and Applications, vol. 24, no. 4, pp. 745-751, April 2006.

[7] A. M. Mathai and S. B. Provost, Quadratic Forms in Random Variables: Theory and Applications. New York, USA: Marcel Dekker Inc., 1992. 